Didáctica. Lengua y literatura

ISSN-e: 1988-2548

https://dx.doi.org/10.5209/dida.65938

\title{
Aprendemos y enseñamos usando el vídeo con estudiantes universitarios de ELE en el ámbito de la Arquitectura y la Construcción
}

\author{
Paloma Úbeda Mansilla ${ }^{1}$; Maria Luisa Escribano Ortega ${ }^{2}$
}

Recibido: 17 de junio de 2017 / Aceptado: 02 de abril de 2019

Resumen. Este artículo presenta una experiencia de investigación realizada en el ámbito del curso Español Académico para la Arquitectura y la Construcción, dirigido a alumnos extranjeros en la Escuela Técnica Superior de Arquitectura de la Universidad Politécnica de Madrid. Los estudiantes participantes presentaban un perfil lingüístico de Usuario Independiente, es decir niveles entre el B1 y B2, según criterios establecidos en el Marco Común Europeo de Referencia para las Lenguas: aprendizaje, enseñanza y evaluación (MCER) recogidos por el Portfolio Europeo de Lenguas Académico y Profesional (ACPEL). Los alumnos crearon, diseñaron y grabaron situaciones de su ámbito académico cotidiano, aprendiendo vocabulario específico y estructuras gramaticales en contextos naturales para ellos, dentro de un discurso de su especialidad. La propuesta fue valorada positivamente por los estudiantes; los videos producidos sirven como referencia para su propio feedback y como herramienta de aprendizaje para animar a otros estudiantes internacionales.

Palabras clave: Español con fines específicos, Oralidad, Actividades Didácticas, Medios audiovisuales, Estudiantes Internacionales, Arquitectura.

\section{[en] Learning and teaching by using task videos activities with university students of ELE in the field of Architecture and Construction}

\begin{abstract}
This article presents a research experience in the field of the Spanish Academic course for Architecture and Construction, aimed at foreign students at the School of Architecture of the Universidad Politécnica de Madrid. The participating students presented a linguistic profile of Independent User, and between B1 and B2 average levels, according to criteria established in the Common European Framework of Reference for Languages: learning, teaching and evaluation (CEFR) gathered by the European Portfolio of Academic Languages and Professional (ACPEL). The students created, designed and recorded situations of their daily academic environment, learning specific vocabulary and grammatical structures in natural contexts for them, within a discourse of their specialty. The proposal was valued positively by the students; the videos produced serve as a reference for their own feedback and as a learning tool to encourage other international students.
\end{abstract}

Keywords: Spanish for specific purposes, Orality, Learning activities, Audiovisual media, International Students, Architecture.

1 Departamento de Lingüística Aplicada a la Ciencia y a la Tecnología

ETS Arquitectura, UPM

paloma.ubeda@upm.es

2 Profesora de Español, PROLINTER-UPM

Universidad Politécnica de Madrid

marialuisa.escribano@upm.es 


\section{[fr] On apprend et on enseigne en utilisant la vidéo avec des étudiants universitaires de ELE dans le cadre de l' Architecture et la Construction.}

Résumé. Cet article presente une expérience de recherche réalisée dans le cadre du cours Espagnol Académique pour l'Architecture et la Construction adressé à des élèves étrangers de l'École Technique Supérieure d'Architecture de 1'Université Politechnique de Madrid. Les étudiants qui y ont participé presentaient un profil linguistique, (utilisateur indépendant) niveau B1-B2, selon les critères établis dans le Cadre Européen Commun de référence pour les langues étrangères: apprendre, enseigner, évaluer (CECR). Ces critères se retrouvent également dans le Portfolio Européen des Langues Académique et Professionnel (PELAP). Les élèves ont créé, conçu et enregistré des situations de leur domaine académique quotidien tout en apprenant du vocabulaire spécifique et des structures grammaticales dans des contextes qui leur sont naturels, dans un discours propre à leur filière. La proposition a été positivement reçue par les étudiants; les vidéos produites servent comme référence pour leur propre feedback et comme outil d'apprentissage pour encourager d'autres étudiants étrangers.

Mots clés: Espagnol avec des fins spécifiques, Oralité, Activités Didactiques, Moyens audiovisuels, Étudiants Internationaux, Architecture.

Sumario: 1. Introducción. 2. Contexto metodológico. 3. Desarrollo de la experiencia. 4. Resultados del cuestionario. 5. Conclusiones. 5.1. Agradecimientos. 6. Bibliografia. 7. Anexo: el cuestionario.

Cómo citar: Úbeda Mansilla, P.; Escribano Ortega, M. L. (2019). Aprendemos y enseñamos usando el vídeo con estudiantes universitarios de ELE en el ámbito de la Arquitectura y la Construcción, Didáctica. Lengua y literatura, 31, 65-78.

\section{Introducción}

A pesar del descenso de la población universitaria y de la proliferación en España de las universidades privadas, el número de estudiantes internacionales que se acercan a la Universidad Politécnica de Madrid (UPM) y concretamente a la Escuela Técnica Superior de Arquitectura de Madrid (ETSAM) se mantiene prácticamente estable desde hace casi 20 años. Los lugares de procedencia y las preferencias académicas de los estudiantes, sin embargo, están en continuo cambio. Si hace dos décadas los estudiantes internacionales que se incorporaban a nuestras aulas procedían casi en su totalidad de universidades europeas, que pretendían tener una experiencia de intercambio en el ámbito universitario con una duración máxima de un curso académico, en la actualidad las situaciones se han diversificado y van desde estudiantes que cursan la carrera completa en nuestra universidad, hasta aquellos que exclusivamente vienen a hacer una investigación, pasando por alumnos de máster, doctorado y una gran gama de estudiantes de intercambio académico con o sin convenios, ahora sí de cualquier parte del mundo.

Limitándonos al análisis de los cuatro últimos cursos académicos podemos observar que la cantidad de universitarios de intercambio es similar, con un ligero incremento de estudiantes procedentes de programas de Erasmus Mundus: 


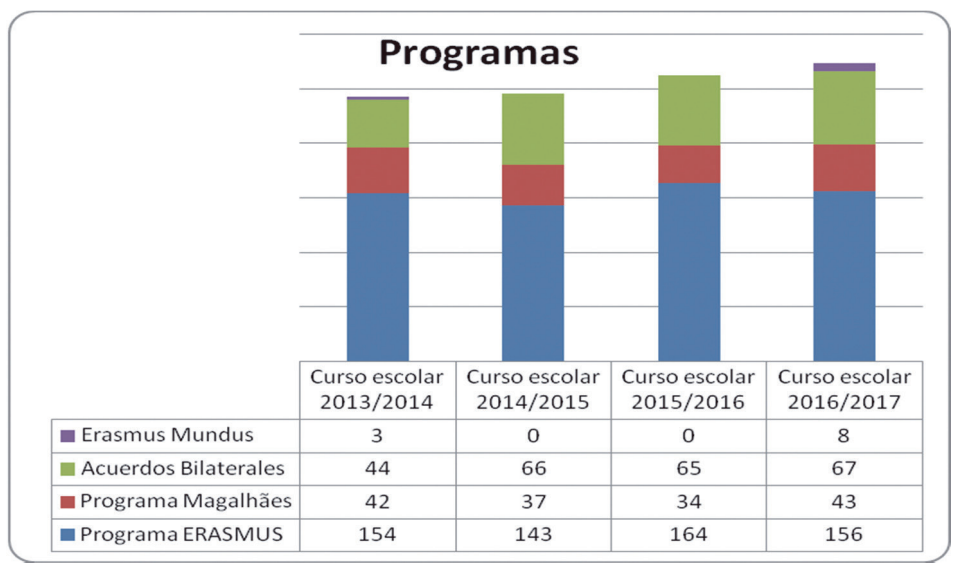

Figura 1. Programas de Intercambio.

En cuanto al nivel lingüístico de los alumnos, hay que destacar el esfuerzo realizado por la Comisión Europea al poner en marcha en 2014 el Online Linguistic Support (OLS), que incluye un examen previo a la experiencia de intercambio, un sistema de autoaprendizaje para mejorar el nivel de lenguas extranjeras del candidato y una posterior evaluación a la finalización del programa. Este sistema ha propiciado la mejora del nivel de español de los universitarios europeos que recibimos.

Diferente es la situación de los estudiantes de fuera de Europa procedentes de los antiguos programas de Erasmus Mundus ${ }^{3}$, a los que no se les exige poseer un nivel mínimo de español. Generalmente estos alumnos y alumnas vienen a colaborar con departamentos o centros de investigación en los que se pueden comunicar en inglés. Su bajo nivel de español determina, en ocasiones, el escaso éxito de la experiencia y, en la mayoría de los casos, la difícil integración con otros estudiantes más allá de su círculo de trabajo habitual.

El cambio sustancial que se está produciendo en estos últimos años es que los estudiantes ya no vienen a España tan solo para experiencias cortas, sino que empiezan a optar por estancias de media-larga duración, es decir, vienen para cursar grado, máster o doctorado completos. Esta proyección expansiva junto al agrandamiento del territorio físico y humano del español se ha recogido en el informe Valor económico del español (García, Alonso y Jiménez, 2012) y hace presagiar un futuro esperanzador que nos obligará a replantearnos las programaciones y los cursos de español como lengua extranjera, ya que la Estrategia de internacionalización de universidades 2015-2020 recomienda ampliar las titulaciones en inglés en las universidades españolas como elemento fundamental de internacionalización.

\section{Contexto metodológico}

Teniendo en cuenta el perfil de los estudiantes y los objetivos de nuestros cursos de Español con Fines Específicos, nos hemos inclinado por un enfoque cognitivo por

3 El programa Erasmus Mundus se ha extinguido y ha pasado a ser una de las acciones que forman parte del Programa Erasmus+. 
lo que tiene de aprendizaje significativo. Este aprendizaje nace de una motivación interna, de valoración de habilidades y destrezas de los aprendientes y de la consideración del estudiante como un sujeto activo, cuyo entorno determina tanto sus percepciones como sus ideas.

Puesto que los alumnos y alumnas pertenecen al colectivo de los estudiantes de arquitectura o de construcción, están inmersos en un entorno conceptual común y comparten una visión artística de la realidad. En definitiva, se sitúan en un plano perceptivo, conceptual y cognitivo similar, que garantizará una óptima adecuación a la propuesta didáctica planteada.

El enfoque basado en la experiencia está totalmente vinculado a la forma que tenemos de usar el lenguaje. El lenguaje, bajo este prisma, no expresa la realidad tal y como es, sino de la manera en que los seres humanos la entienden y en la que conceptualizan los objetos y situaciones del mundo exterior. En el caso de la Arquitectura, como Forty (2000) nos confirma, las palabras que se usan describen algo más que ladrillos y cemento e indican la forma en que entendemos y convivimos con los edificios. Tenemos en cuenta, por tanto, que las actividades en las que se dice y se realiza algo son las que tienen mayor índice de retención en la memoria de los estudiantes; como recuerda Alonso, "los alumnos retienen el 90\% de lo que se dice y luego se realiza" (Alonso, 2000, p. 189). Por ello, entre las actividades propuestas, destacan aquellas relacionadas con la simulación (role-play) que consiguen motivar y transmitir ciertas experiencias que todavía no poseen los estudiantes, porque están orientadas a su futuro entorno profesional. La incorporación del uso de metáforas en las actividades de simulación potencia todavía más el proceso de aprendizaje. Las actividades de simulación escogidas corresponden a diferentes situaciones en las que el arquitecto puede verse involucrado, siguiendo la propuesta de Yalden: "a task should be realistic; it should be something the learner will do in the target language" (1986, p. 152).

El contexto en que se enmarca la actividad que proponemos para satisfacer las exigencias específicas de una enseñanza de Español con Fines Específicos (ESP) y al mismo tiempo recrear una experiencia real, se va a basar en el uso de:

- Nuestra propia experiencia en el entorno arquitectónico profesional, adquirida a través de contactos regulares y colaboraciones con Profesores y ex alumnos de la Escuela Técnica Superior de Arquitectura de Madrid (ETSAM), y de la observación en diferentes estudios de arquitectura españoles.

- El análisis de necesidades para este perfil de estudiantes, llevado a cabo en el ámbito académico.

- Los escenarios en los que los estudiantes internacionales deben desenvolverse en su vida académica.

- Materiales de gramática y léxico que reflejan las situaciones académicas en las que los estudiantes se encuentran involucrados.

El desarrollo de esta experiencia tiene profundas raíces cognitivas, con el objetivo de facilitar la asimilación y aprendizaje del idioma, y considera tanto los particulares procesos cognitivos, como el estilo de aprendizaje dominante del colectivo.

Para aprovechar mejor este enfoque, la propuesta considera complicar gradualmente la complejidad de las actividades en el tiempo, tanto en lo que se refiere a los procesos activos donde los estudiantes son protagonistas (búsquedas en Internet, actividades de simulación,...) como al contenido de los textos, y a la progresiva in- 
tegración de las cuatro destrezas lingüísticas a lo largo de las asignaturas de español. Como comenta Robinson:

...tasks making increasing conceptual/communicative demands increasingly engage cognitive resources, which progressively exploit learning mechanisms leading to greater analysis, modification and reestructuring of interlanguage, with consequent performance effects. (2001, pp. 301-302)

Para ello, la experiencia de esta actividad audiovisual que se presenta en este artículo se va a fundamentar en el uso de cuatro elementos, que utilizados independientemente o de forma integrada, y con complejidad progresivamente creciente, desarrollen las cuatro destrezas lingüísticas básicas: lectura, escritura, comprensión oral, expresión oral. Todo ello dentro de un contexto profesional arquitectónico "recreado" en el aula que represente la "experiencia", y adaptándose de forma natural a los procesos cognitivos y los estilos de aprendizaje propios del colectivo. José Antonio Marina nos habla de la ciencia cognitiva como aquella en la que convergen «muchos saberes dispersos, procedentes de muchas disciplinas [como son] la filosofía, la psicología genética, la psiconeurología, la inteligencia artificial» (1993, p. 241).

La interdisciplinariedad a la que nos hemos referido antes también viene reflejada en las palabras de Langacker, que asegura que:

The primary need of linguistics today ... is a conceptual framework ... which permits the unified description of the many facets of language structure that present theories insist on forcing into separate boxes. (1987, p. 31)

A diferencia de otras gramáticas, que hablaban de dicotomías como sintaxissemántica o categoría-función, la lingüística cognitiva es un modelo basado en el uso. La función de la lengua es importante. Como señalan Cuenca \& Hilferty, "los lingüistas cognitivos consideran que, en general, la función y el significado condicionan la forma y no al contrario" (1999, p. 29). También se refiere la gramática cognitiva a la lengua real, y no a una idealización de la lengua, que también habrá que tener en cuenta a la hora de aplicarse a la enseñanza de una segunda lengua.

El cognitivismo se plantea estudiar todos los aspectos del lenguaje, dando una explicación conjunta a los aspectos generales y a los aspectos específicos de cada lengua. En palabras de Langacker (1987), "el lenguaje es una mezcla de regularidad e irregularidad; por eso hay que tratar todo lo que nos encontremos de forma natural".

Resumiendo, diremos que la gramática cognitiva está interesada, sobre todo, en la función, el significado y el uso del lenguaje, aunque por supuesto, también considera la forma. De ahí que tengamos que tener en cuenta todos estos factores a la hora de enseñar una lengua. Por eso, igual que se da una unión entre forma y significado/ función/uso, no se pueden aislar los procesos cognitivos que se dan en la mente del estudiante de una segunda lengua, porque también se ha estudiado el proceso de adquisición de una segunda lengua como resultado de procesos cognitivos generales (McLaughlin, 1987; McLeod \& McLaughlin, 1986).

Así pues, la experiencia que presentamos se enmarque en lo que conocemos como una actividad audiovisual llevada a cabo por unos alumnos en un ámbito de la enseñanza del español con Fines Específicos. Fontanet, et alt. (2008) hace referencia a las últimas prácticas y experiencias llevadas a cabo en el ámbito universitario. 
Pombo (2011) recoge las necesidades y pautas para un diseño de curso específico para estudiantes de arquitectura internacionales ELE en el ámbito de la arquitectura. Si a esto añadimos que los estudiantes de arquitectura tienen un estilo de aprendizaje predominantemente cinestésico (Úbeda \& Escribano, 2002) y que por tanto les gusta aprender by doing, la motivación del grupo está asegurada.

De este modo, se les propone tomar la responsabilidad de su propio aprendizaje, apoyado en el vídeo pero con el objetivo de desarrollar sus destrezas orales en el estudio de la lengua española. Johnson, K. E. Golombek, P.R. (2016) aluden al proceso de aprendizaje a partir de la actividad en la cual uno se encuentra involucrado y de la importancia del papel del profesor en participar con el grupo. En este caso, nuestro grupo de estudiantes internacionales que estudian español en un contexto académico en la escuela de arquitectura de Madrid.

\section{Desarrollo de la experiencia}

Los participantes de esta actividad fueron 24 estudiantes internacionales matriculados en el Curso Especial de Español Académico para la Arquitectura y la Construcción. La asignatura es de 3 ECTS (European Credit Transfer System) y se ofrece a todos los estudiantes internacionales de la UPM a partir de un nivel B1 del MCER. Las clases tuvieron lugar dos días a la semana, en sesiones de dos horas, con un total de 20 sesiones, es decir 40 horas de clase, a lo largo de aproximadamente 14 semanas. Los estudiantes que eligieron esta asignatura procedían de las titulaciones asociadas al campo de la arquitectura y construcción y la media de edad de los participantes era de 23 años. El grupo estaba formado por 13 mujeres y 11 hombres. Las nacionalidades de los alumnos y alumnas, que formaron parte de esta experiencia, se recogen en el siguiente gráfico:

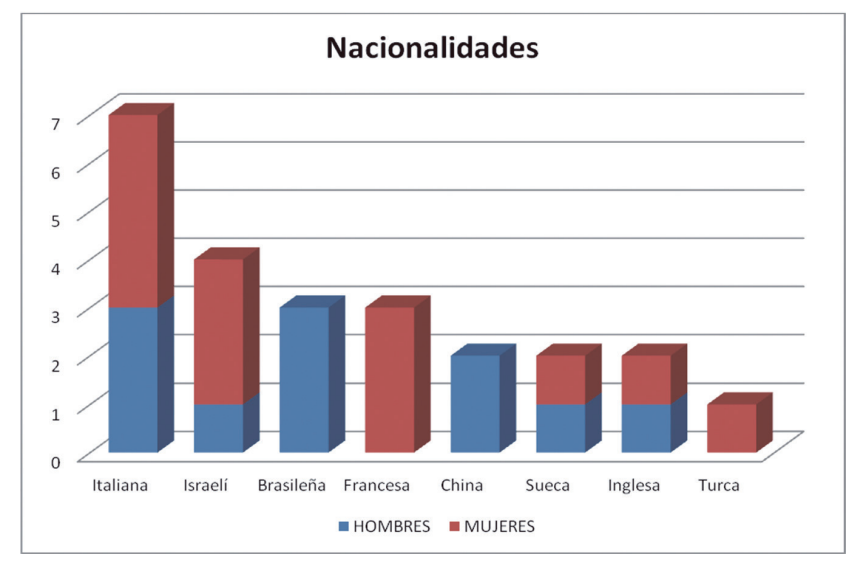

Figura 2. Nacionalidades.

Como fase previa preparatoria se propuso a los estudiantes que formaran grupos de trabajo en la clase. Se formaron cuatro grupos de seis estudiantes, cubriendo el total de los 24. El planteamiento fue cubrir estos dos objetivos: 
- Fomentar el trabajo colaborativo de los estudiantes enriqueciendo los vínculos de relaciones personales del grupo.

- Reforzar a la vez la autonomía de aprendizaje del alumno en la última etapa con la memorización de un papel para interpretar, con el objetivo de aumentar la autoestima y la seguridad de los estudiantes.

Ambos objetivos no solo serían válidos para el resto de las asignaturas de la Escuela, sino también para ser utilizados en un entorno real del futuro ámbito laboral de estos estudiantes. Paralelamente esta forma de trabajo reforzaba las estrategias cognitivas que el grupo posee y que mencionamos en el apartado anterior.

El ejercicio se planteó como un proyecto colaborativo, siguiendo los principios de interdependencia positiva, responsabilidad individual y grupal, interacción estimuladora, desarrollo de competencias de trabajo en equipo y evaluación grupal (Johnson \& Johnson, 1999). Los cuatro grupos de estudiantes plantearían una serie de situaciones en el entorno académico, cada grupo elegiría dos para trabajar con más profundidad y, a partir de aquí, cada grupo crearía una serie de diálogos prototípicos de estas dos situaciones cotidianas elegidas. Posteriormente los estudiantes grabarían en vídeo estas situaciones a partir de los guiones para los diálogos que ellos mismo elaboraron.

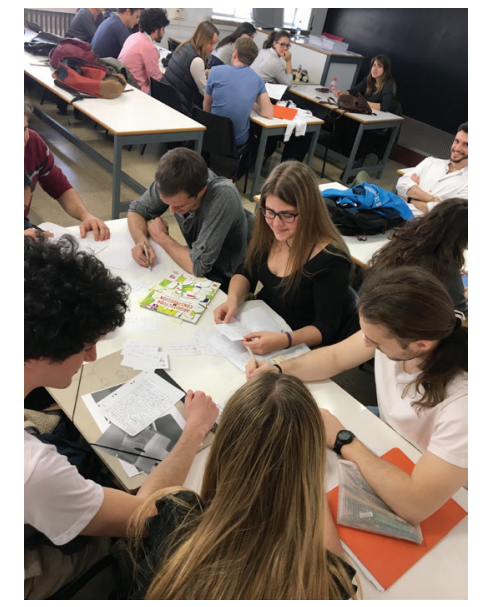

Imagen 1. Grupos de estudiantes trabajando.

Este diálogo debía reflejar la realidad de una experiencia y/o las necesidades que el estudiante podía tener bajo cada paraguas situacional elegido. Cada grupo fue responsable de generar una conversación de unas 300 palabras aproximadamente en español teniendo en cuenta las funciones comunicativas de niveles B1-B2 sugeridas por el profesor:

- Pedir y dar información

- Pedir confirmación

- Confirmar información previa

- Dar y pedir opiniones

- Valorar

- Pedir aprobación
- Expresar acuerdo o desacuerdo

- Presentar un contraargumento

- Expresar certeza o evidencia

- Expresar posibilidad

- Expresar obligación y necesidad

- Expresar conocimiento de algo 
Cada texto-diálogo realizado en clase por el grupo fue supervisado por el profesor y las correcciones y explicaciones se iban realizando a medida que surgían las dudas. El profesor por tanto iba de grupo en grupo dedicando una media de veinte minutos por grupo en cada sesión. Los errores más significativos que se detectaron a la hora de realizar estos diálogos fueron problemas de vocabulario, de uso de tiempos de pasado, uso del subjuntivo, concordancias sintácticas, preposiciones, uso de ser y estar, etc.

El proceso de creación de diálogos se realizó en dos sesiones. Una vez terminados y corregidos se fotocopiaron y se intercambiaron entre los otros grupos para trabajar las estructuras de gramática y de vocabulario nuevo o vocabulario específico de arquitectura. Se pidió a los estudiantes que trabajaran con tres colores diferentes (Úbeda \& Escribano, 2008). Cada color tenía una finalidad: rojo desconocido, azul vocabulario específico, amarillo la función comunicativa que se trabajaba.

\section{En el taller de maquetas}

Personajes: Estudiante, Profesor ayudante. (Taller de maquetas. Los estudiantes trabajan en silencio mientras el profesor se va pasando por las mesas. Un alumno intercepta al profesor).

E: Hola qué tal. ¿Podrías decirme qué materiales puedo utilizar para la maqueta?

P: Hola. Sí, bueno, los materiales... son los que ves aquí. Tenemos espuma rígida, madera, cartulina, cartón, lijas, pegamento... También puedes utilizar, si lo deseas, tintura. Te recomiendo utilizar el algodón y las ramas secas para los árboles de la parcela, que da buenos resultados.

E: Ah, vale. Y en cuanto a herramientas, ¿cuáles hay disponibles?

P: Aquí en el taller tenemos cuchillas, reglas, seguetas, una cortadora... Tienes que tener cuidado de no hacerte daño y acordarte, eso sí, de devolverlas cuando hayas terminado de usarlas, ya que los demás también las necesitan y no tenemos demasiadas.

E: Y, ¿Qué pasos hay que seguir para hacer la maqueta?

P: Pasos, pasos... No hay unos pasos definidos, hombre jaja, simplemente acuérdate que, además de escoger bien los materiales, hay que ser limpio y preciso a la hora de medir, cortar, lijar, pegar... no hacerlo deprisa y corriendo porque luego salen chapuzas.

E: Jeje, De acuerdo. Una última pregunta. ¿Es verdad que todos tenemos que usar escala 1/500?

P: Cooorrecto. Los materiales son más libres, pero la escala debe ser 1/500 para todos. Tienes las curvas de nivel del solar a esa escala en el archivo de Autocad que os hemos enviado al mail.

E: ¿Quieres decir que todo lo demás lo elegimos nosotros?

P: Sí, claro, no te voy a hacer yo el proyecto. Los llenos, vacíos, vanos, colores... todo eso ya depende de cada uno.

E: Vaaale. Gracias. Voy a ello / me pongo a ello

Los diálogos partían de un nivel de lengua de nivel B1 y recogían algunos de los descriptores fijados por el profesor para los grupos que pusieran en práctica lo recogido en el ACPEL Portfolio (2009). Durante esta dinámica el papel del profesor era asegurarse de que cada grupo ponía en práctica los descriptores fijados por el nivel que los alumnos debían adquirir.

Estos diálogos serían la base de los guiones que los estudiantes debían aprender y representar delante de las cámaras como tarea final del curso. Una vez que los diálo- 
gos estuvieron listos, cada grupo decidió representar los que había creado y entre los repetidos se eligió uno. En cada grupo se decidieron los papeles de cada estudiante, y cada uno memorizó su guion.

En la imagen siguiente se recoge un momento de repaso de los guiones antes de ser representados.

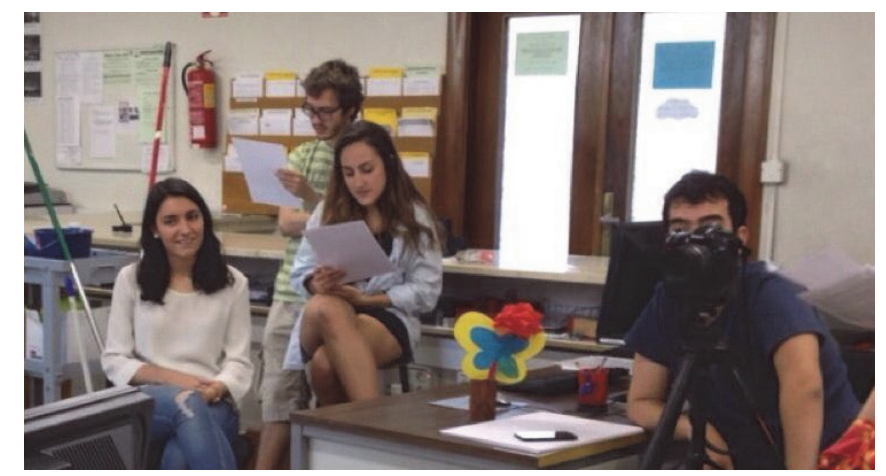

Imagen 2. Grupo de estudiantes.

El proceso de grabación de cada diálogo se llevó a cabo a lo largo de las dos horas de clase. El número de repeticiones fue muy variable de una sesión a otra. Los estudiantes estaban presentes todos los días, aunque no les tocara actuar. Más ejemplos de los diálogos ya corregidos, y que sirvieron de guion para los videos que produjeron, pueden verse en http://aplai2.aq.upm.es/typologies.html y en el canal youtube educativo de la de la UPM: https://www.youtube.com/watch?v=t9KxfiyFvTI\&list= PL8bSwVy8_IcMp3x4L1RYZHcn6gNXbVUo1.

\section{Resultados del cuestionario}

Al finalizar el curso se preparó un cuestionario para conocer cuan satisfactorio había sido el aprendizaje de esta actividad con los estudiantes internacionales que escogen esta asignatura de Español con Fines Específicos en el campo académico de la arquitectura y construcción. Aunque la muestra fue muy pequeña nos serviría como estudio piloto para poder continuar trabajando en cursos posteriores, ya que esta asignatura se imparte cuatrimestralmente dos veces al año durante un curso académico. Los resultados fueron los siguientes:

Los 24 estudiantes que participaron en el curso, y por tanto en esta experiencia global piloto, constataron que el proceso de grabación fue lo más atractivo de la experiencia. Escribir el diálogo y aprender estructuras y vocabulario se consideró una tarea más tediosa. El trabajo de representar lo escrito no supuso un problema para ellos.

En la Fig. 3 se valoran los tres procesos a los que hemos aludido:

1. a. Escribir una conversación en un contexto académico.

1. b. Representar oralmente lo previamente escrito

1. c. Grabar en vídeo el diálogo producido en grupo por los alumnos. 


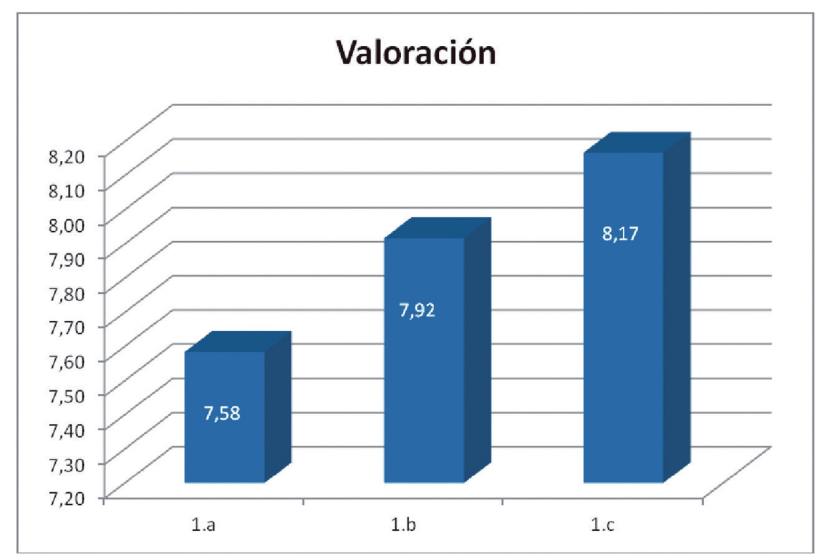

Figura 3. Valoración de los estudiantes.

Las valoración general de todas las preguntas del cuestionario es positiva tal y como se puede ver en la Fig. 4. Los estudiantes señalan que les ha gustado trabajar de esta forma y que no les importaría repetir la experiencia en un futuro. Curiosamente, los estudiantes no recomiendan este tipo de experiencias para otras asignaturas de su titulación (quizás por las características de las asignaturas técnicas). Las preguntas del cuestionario se recogen en el anexo, al final de este artículo.

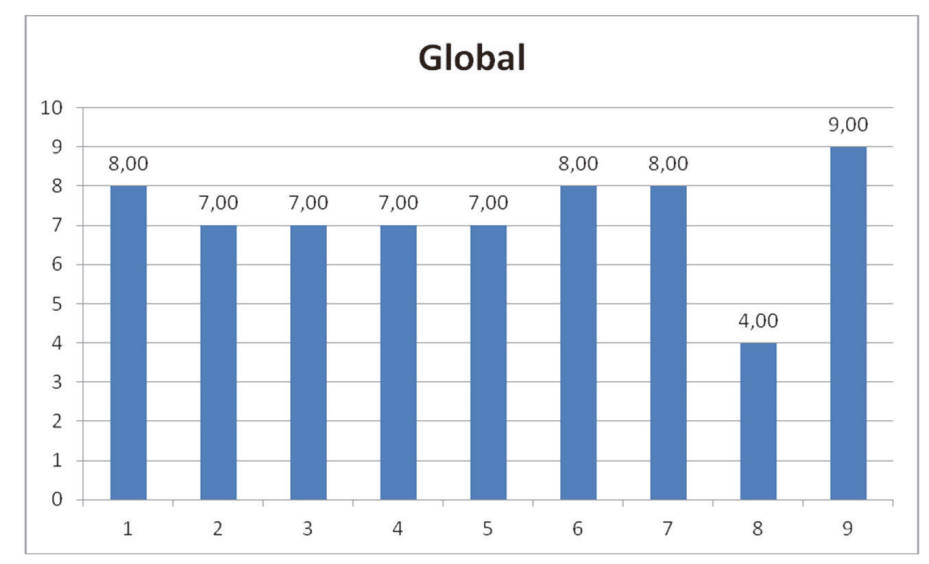

Figura 4. Las preguntas globales de todo el cuestionario.

Si segregamos las valoraciones de las respuestas por género (Fig. 5), en el que $H$ son hombres y $M$, mujeres, no se observa una información relevante, a excepción de dos aspectos:

- la 1, 2, y 3 en las que las mujeres dan una puntación ligeramente superior, sugiriendo que les ha gustado más el enfoque, quizás por su alto contenido de interacción social. 
- la 4 y la 6, ligeramente inferior, sugiere que parecen no haber afianzado tan bien las estructuras gramaticales, ni adquirido tanta seguridad como los hombres (o su nivel de auto-exigencia es mayor).

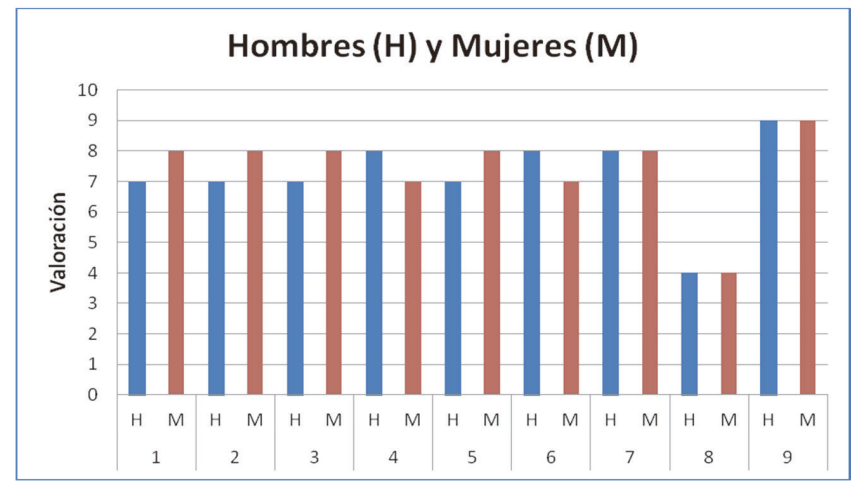

Figura 5. Respuestas por género.

Finalmente, en el histograma representado en la Fig. 6 se recogen las respuestas proporcionadas por los estudiantes para mostrar cuántas respuestas supusieron una puntuación u otra en función de la pregunta y el grupo correspondiente $(\mathrm{H} / \mathrm{M})$. Por ejemplo, las barras correspondientes a 4-ELE-M indican cuántos alumnos (no alumnas) dieron cada valoración (de 1 a 10) a la pregunta 4: de los 13 estudiantes, 3 dieron una valoración de 7, 8 una valoración de 8 , y 2 una valoración de 9.

En el siguiente gráfico se aprecia la concentración de respuestas en los valores 8 y 9, lo cual confirma la utilidad de este tipo de enfoques en la enseñanza de lenguas que ya se había deducido a partir de la figura 3. Sin embargo, llaman la atención los dos picos en la valoración 5; estos picos corresponden a la pregunta 8 , para $\mathrm{H}$ (7 alumnos) y M (8 alumnas). Esto indica como ya se ha mencionado anteriormente que los estudiantes de un perfil científico/técnico no apuestan porque esta metodología, que consideran adecuada para el aprendizaje de español, pueda ser aplicada en otras asignaturas de su carrera.

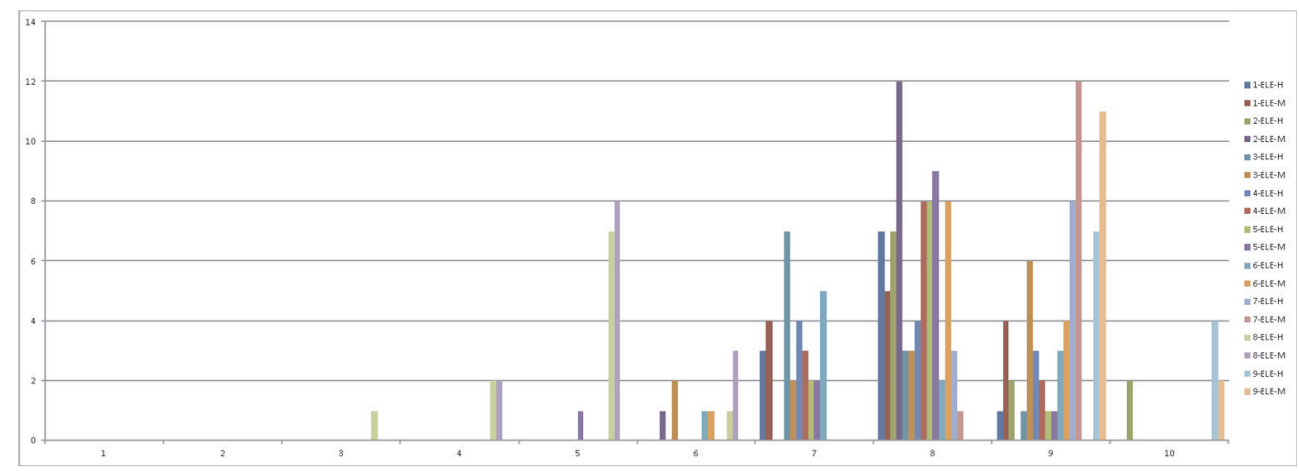

Figura 6. Histograma de la evaluación de los estudiantes. 


\section{Conclusiones}

Es evidente que las nuevas generaciones de estudiantes necesitan actividades en las que se sientan involucrados desde el principio al fin para no perder la motivación.

- Los estudiantes crearon, diseñaron y grabaron situaciones de su ámbito académico cotidiano, aprendiendo vocabulario específico y estructuras gramaticales en contextos naturales para ellos, dentro de un discurso de especialidad.

- Los estudiantes con esta experiencia se responsabilizan de su propio aprendizaje ya que, con la grabación de la situación creada en video, pueden desarrollar su destreza oral en ELE.

- Los videos grabados sirven como referencia para su propia retroalimentación y como herramienta de aprendizaje para animar a otros estudiantes internacionales. El aprendizaje es duradero y se recuerda.

Este tipo de actividad apoya la idea de Wan Ng (2015):

...there has been a fast pace of change in the evolution of digital technologies that are potentially useful for education. If we also add students are skillful with the technologies tools, it also increases students' self-confidence in term of foreign language communication. By using these media gadgets, communication is developed in a natural environment that enhances classroom learning in a positive and engaging way.

Hoy el rol del docente ha cambiado, debemos enseñar al alumno cómo trabajar con la información, cómo trabajar en equipo, cómo presentar los datos que tiene y cómo hacer de las capacidades del grupo una herramienta metodológica útil para usar en el aula.

\subsection{Agradecimientos}

Gracias a todos los estudiantes del curso Español para la Arquitectura y Construcción que formaron parte de esta experiencia audiovisual educativa.

\section{Bibliografia}

Alonso, C. (2000). Aprendizaje y ordenador. Madrid: Dykinson.

Cuenca \& Hiffelty (1999). Introducción a la lingüistica cognitiva. Madrid: Grupo Planeta.

Duran, P. Et alt. (2001). ACPEL Portfolio (Academic and Professional ELP) for Higher Ecucation and Professional Language Learners Purposes, Ed. Edymion S.L.

Escribano Ortega, M.L. \& Úbeda Mansilla, P. (2002). Entre el autodidactismo y los fines especifico: Trabajo por dossier como alternativa en grupos multidisciplinares. Comunicación y pedagogía: Nuevas tecnologías y recursos didácticos, ISSN 1136-7733, $\mathrm{N}^{\circ} 180$.

Estrategia de Internacionalización de las universidades 2015-2020 (en línea). http://www. mecd.gob.es/educacion-mecd/dms/mecd/educacion-mecd/areas-educacion/universidades/politica-internacional/estrategia-internacionalizacion/EstrategiaInternacionalizaci-nFinal.pdf [Consulta 1 de junio de 2017] 
Fortanet-Gómez, Christine A. Räisänen (2008). ESP in European Higher Education: Integrating Language and Content. John Benjamins.

Forty, A. (2000). Words and Buildings: A Vocabulary of Modern Arquitecture. London Thames \&Hudson.

García, ALONSO y JIMENEZ (2012). Valor Económico del Español, Fundación Telefónica Valor Económico del Español (en línea) https://www.cervantes.es/imagenes/file/biblioteca/valor_economico_espanol/valor_economico_espanol.pdf

http://www.fundaciontelefonica.com/arte_cultura/publicaciones-listado/pagina-item-publicaciones/itempubli/183/ [Consulta 1 de junio de 2017]

Gromik, N.A (2011). Cell phone video recording feature as a language learning tool: A case study. Computer and Education. Elsevier.

Johnson, K.E. \& Golombek, P.R. (2010). (Eds.). Research on Second Language Teacher Education: A Sociocultural Perspective on Cultivating Teachers' Professional Development ESL \& Applied Linguistics Professional Series. London \& New York. Routledge.

Johnson, J.E. \& Golombek, P.R. (2016). Mindfull L2 Teacher Education. New York. NY: Routledge.

Johnson. D.W. \& Johnson R.T. (1999). Making Cooperative Learning Work. Taylor \& Francis Group.

Jhonson, D, Jhonson, R. \& Hulebec, E.J. (1994). El aprendizaje cooperativo. Barcelona: Paidós.

Langacker, R.W. (1987). Foundation of Cognivive Grammar. Vol. I: Theoretical Presequisites. Stanford University Press.

Marco Común Europeo de Referencia para las Lenguas: aprendizaje, enseñanza, evaluación. Consejo de Europa (2002) (en línea) http://cvc.cervantes.es/ensenanza/biblioteca ele/marco/cvc_mer.pdf [Consulta 1 de junio de 2017]

Marina, J.A. (1993). Teoría de la Inteligencia creadora. Barcelona: Anagrama.

Pombo I. (2011). Curso de ELE para estudiantes de arquitectura ARQUI_ELE Análisis de necesidades y pautas para su diseño. Memoria de Máster. Madrid: Nebrija Universidad.

Robinson, K. (2001). Out of Our Minds: Learning to be creative. Capstone Publishing.

Romero Tena, R. (2001). “Utilización didáctica del Video" (en línea) http://tecnologiaedu. us.es/revistaslibros/public8.htm

Shanahan, L., Ortlieb, E. \& McVee, M. (2015). Video Research in Disciplinary. Emerald Group Publishing Literacies.

Úbeda Mansilla, P. \& Escribano Ortega, Ma L. (2002). "Estudio contrastivo de los estilos de aprendizaje en los estudiantes de arquitectura". Didáctica (Lengua y Literatura), 14, 251271.

Wai Meng Chan, Kwee Nyet Chin, Masanori Nagami \& Titima Suthiwan (2011). Media in Foreign Language Teaching and Learning. Walter de Gruyter.

Wan NG (2015). New Digital Technology in Education: Conceptualizing Professional Learning for Educators. Springer.

Yaden, J. (1987). Principles of Course Design for Language Teaching. Cambridge: UniversityPress. 


\section{Anexo: el cuestionario}

ELE/ESL CUESTIONARIO JUNIO 2015

Hombre:

Mujer:

Edad:

1. Me ha gustado la experiencia de trabajar el uso de una lengua extranjera creando unos guiones situacionales que se fueran a grabar en video.

$\begin{array}{llllllllll}-1 & 2 & 3 & 4 & 5 & 6 & 7 & 8 & 9 & 10+\end{array}$

Valora cada uno de los siguientes aspectos en función de cuánto crees que te han aportado en el aprendizaje.

1. a. El proceso de crear un dialogo en un contexto habitual de comunicación académica y profesional.

$$
\begin{array}{llllllllll}
-1 & 2 & 3 & 4 & 5 & 6 & 7 & 8 & 9 & 10+
\end{array}
$$

1. b. El representar lo aprendido de forma oral de una forma cercana a un futuro próximo de uso.

$$
\begin{array}{llllllllll}
-1 & 2 & 3 & 4 & 5 & 6 & 7 & 8 & 9 & 10+
\end{array}
$$

1. c. El proceso de grabación.

$$
\begin{array}{llllllllll}
-1 & 2 & 3 & 4 & 5 & 6 & 7 & 8 & 9 & 10+
\end{array}
$$

2. Considero que he aprendido de una forma más natural que como lo he hecho hasta ahora.

$$
\begin{array}{llllllllll}
-1 & 2 & 3 & 4 & 5 & 6 & 7 & 8 & 9 & 10+
\end{array}
$$

3. Estoy muy satisfecho con relación al aprendizaje que he adquirido con esta experiencia.

$$
\begin{array}{llllllllll}
-1 & 2 & 3 & 4 & 5 & 6 & 7 & 8 & 9 & 10+
\end{array}
$$

4. He fijado las estructuras gramaticales de la lengua de una forma más natural.

$$
\begin{array}{llllllllll}
-1 & 2 & 3 & 4 & 5 & 6 & 7 & 8 & 9 & 10+
\end{array}
$$

5. He aprendido el vocabulario en un contexto más útil y lo recuerdo más fácilmente.

$$
\begin{array}{llllllllll}
-1 & 2 & 3 & 4 & 5 & 6 & 7 & 8 & 9 & 10+
\end{array}
$$

6. He adquirido mucha seguridad en el uso de la lengua extranjera aprendida con esta experiencia.
1
23
45
$6 \quad 7$
89
10

7. Me gustaría repetir otra experiencia similar en un futuro.
$-1$
3
456
$6 \quad 7$
89
$10+$

8. Recomiendo estas prácticas docentes en otras asignaturas de mi carrera.
4
$\begin{array}{llll}6 & 7 & 8 & 9\end{array}$
$10+$

9. Creo que este tipo de experiencias es muy útil en el uso de lenguas extranjeras.

$\begin{array}{llllllllll}-1 & 2 & 3 & 4 & 5 & 6 & 7 & 8 & 9 & 10+\end{array}$ 